\title{
Association of H3K4me3 and CBX7 Levels to the Down-Regulation of GKN1 Gene Expression in Gastric Cancer Cells
}

\author{
Chiara Stella Di Stadio ${ }^{*}$, Filomena Altieri ${ }^{*}$, Antonella Federico ${ }^{1 *}$, Giuseppina Miselli1, \\ Maurizio Grillo², Emilia Rippa"\#, Paolo Arcari1,3\# \\ ${ }^{1}$ Department of Molecular Medicine and Medical Biotechnology, University of Naples Federico II, Naples, Italy \\ ${ }^{2}$ Department of Surgery, Antonio Cardarelli Hospital, Naples, Italy \\ ${ }^{3}$ CEINGE, Advanced Biotechnology Scarl, Naples, Italy \\ Email: "emilia.rippa@unina.it, "arcari@unina.it
}

How to cite this paper: Di Stadio, C.S., Altieri, F., Federico, A., Miselli, G., Grillo, M., Rippa, E. and Arcari, P. (2019) Association of H3K4me3 and CBX7 Levels to the Down-Regulation of GKN1 Gene Expression in Gastric Cancer Cells. Journal of Biosciences and Medicines, 7, 146-156. https://doi.org/10.4236/jbm.2019.75016

Received: February 13, 2019

Accepted: May 18, 2019

Published: May 21, 2019

Copyright $\odot 2019$ by author(s) and Scientific Research Publishing Inc. This work is licensed under the Creative Commons Attribution International License (CC BY 4.0).

http://creativecommons.org/licenses/by/4.0/

(c) $\underset{\mathrm{EY}}{\mathrm{EY}}$ Open Access

\begin{abstract}
Gastrokine $1(G K N 1)$ is a highly secreted gastric mucosal protein in normal individuals but strongly down-regulated or totally absent in gastric cancer subjects. An epigenetic mechanism might be responsible for GKNl gene silencing probably through the activity of a transcription factor in association with the enzymes SUV39H1 and HDACs on the GKN1 promoter. In fact, compared to non-tumor tissues, a high increase of H3K9me3 level was observed in the corresponding tumor ones. Because H3K4me3 seems to be a possible epigenetic mark for active euchromatin, we try to verify the $\mathrm{H} 3 \mathrm{~K} 4 \mathrm{me} 3$ level on the $G K N 1$ promoter in gastric cancer tumor specimens. In addition, we also attempt to highlight if $\mathrm{CBX} 7$ could be the possible regulatory transcription factor correlated to $G K N 1$ gene promoter. Therefore, we evaluated if the CBX7 expression levels could be associated with GKN1 down-regulation in gastric cancer. To this purpose, 2 pairs of non-tumor and tumor surgical specimens from patients with gastric cancer were analyzed for H3K4me3 by chromatin immunoprecipitation (ChiP) assays, and 9 pairs were instead analyzed by Western blotting for GKN1, and CBX7 expression levels, respectively. The results suggested that the observed increase of $\mathrm{H} 3 \mathrm{~K} 4 \mathrm{me} 3$ in tumor samples was not in agreement with its proposed function whereas the expression of $\mathrm{CBX} 7$ was not associated with the down-regulation of GKN1. In particular, the expression levels of CBX7 in tumor samples might suggest a survival role in gastric cancer.
\end{abstract}

\section{Keywords}

CBX7, Gastrokine 1, Gastric Cancer, Epigenetics, H3K4 Methylation

*Equally contributed to the work. 


\section{Introduction}

Gastrokine $1(G K N 1)$ is an $18 \mathrm{kDa}$ stomach-specific protein that plays an important role for the physiological function of the gastric mucosa, for the renewal of the epithelial cell layer and in maintaining the integrity of the gastric mucosa. The down-regulation of the protein is associated with an increased risk of gastric cancer development. The protein is, in fact, down-regulated in Helicobacter pylori-infected gastric tissues and its expression is almost detected or completely lost in gastric cancer tissues and precancerous lesions [1] [2]. So it is clear that the expression of GKN1 decreased throughout the progressive stages of neoplastic transformation, from gastritis to intestinal metaplasia, and that it plays an important role in the process of formation and development of gastric cancer. It has also been demonstrated that $G K N 1$ expression induces apoptosis in gastric cancer cells [3]. This finding was also confirmed by the ability of recombinant human $G K N 1$ (rhGKN1) to exert a higher antiproliferative effect on gastric cancer cells (AGS) compared to normal human embryonic kidney cells (HEK 293) and non-gastric cancer cells (H1355) [4]. The higher sensitivity of AGS cells to $G K N 1$ exposure was likely linked to the role played by the protein in maintaining gastric mucosal integrity and to its function as a gastric tumor suppressor.

Regarding the mechanism by which $G K N 1$ gene is down-regulated in gastric cancer, there still remains an open question even though epigenetic mechanisms seem to be involved in this process. In fact, we showed for the first time a possible mechanism of histone modifications that could lead to dysregulate GKN1 gene transcription in gastric cancer. We have found the massive presence of the repressive histone modification $\mathrm{H} 3 \mathrm{~K} 9 \mathrm{me} 3$ (histone 3 trimethylation at lysine 9) and the recruitment of the specific histone methyltransferase SUV39H1 (histone-lysine N-methyltransferase) on the GKN1 gene promoter in human tumor tissues with respect to normal tissues [5]. Under this regard, both histone modification H3K4me2t (histone 3 dimethylation at lysine 4) and H3K4me3 (histone 3 trimethylation at lysine 4 ) are proposed to be epigenetic marks. In particular, H3K4me3 is associated with functional or open chromatin [6] [7] whereas H3K9me2 is a mark linked with silenced/repressed euchromatin instead [8] [9]. Therefore, H3K4 methylations levels could be indicative of chromatin in a poised state [7].

Polycomb group ( $\mathrm{PcG}$ ) proteins act at different chromosomal sites by forming a complex, the polycomb repressive complexes (PRC) leading to the transcription repression of gene expression through an epigenetic mechanism [10]. Chromobox homolog 7 (CBX7) is a member of PcG family proteins that act in an independent manner during the progression of several types of cancer [11] [12] [13] [14] [15]. The association between the absence of CBX7 and the appearance of a highly malignant phenotype seems to be an oncologic characteristic. In fact, CBX7 was found overexpressed in gastric cancer cell lines and gastric tumors and correlated with clinical stage, patients' age, and lymph node metas- 
tasis [14] [16]. Loss of CBX7 expression in gastric cancer cells led to increased cellular senescence decreased cellular proliferation and migration ability [14]. However, the role of CBX7 in cancer development is still not clear since it has been demonstrated that it may behave as a tumor suppressor or act as an oncogene in relation to cancer type and cellular context [11] [12] [13] [14] [15].

In this work, we focus on trimethylation of $\mathrm{H} 3 \mathrm{~K} 4(\mathrm{H} 3 \mathrm{~K} 4 \mathrm{me} 3)$ as a possible epigenetic mark associated with $G K N 1$ down-regulation and tried to highlight if CBX7 expression at protein level was also associated to GKN1 behavior in gastric cancer tissues.

\section{Materials and Methods}

\subsection{Materials}

Mouse GKN1 monoclonal antibody (M01), clone 2E5, was purchased from Abnova (Taipei, Taiwan). Rabbit polyclonal CBX7 (P-15) antibody [sc-70232] was from Santa Cruz Biotechnology (Dallas, TX, USA).

\subsection{Cell Cultures, Human Tissues, and Western Blotting}

Human gastric adenocarcinoma cell line (AGS) was grown in DMEM-F12 supplemented with heat-inactivated FBS, $1 \%$ penicillin/streptomycin and $1 \%$ L-glutamine at $37^{\circ} \mathrm{C}$ in a $5 \% \mathrm{CO}_{2}$ atmosphere.

Human GC tissues were from our collection of specimens as reported [17]. All patients were interviewed for their habit (alcohol intake, chronic use of drugs, smoking dependency). During surgery, Hospital Pathologist performed the dissection of non-tumor $(\mathrm{N})$ and tumor tissues $(\mathrm{T})$ and characterized the non-tumor gastric mucosa from the comparison between the macroscopic aspects of normal adjacent tissue versus the tumor one [18]. GC was staged and graded according to the American Joint Committee on Cancer criteria [19]. This study was approved by the University of Naples Federico II Ethics Committee (Comitato Etico Università Federico II) protocol number 34/15. All western blots were performed on $20 \mu \mathrm{g}$ of cell extracts using mouse anti-GKN1 at 1:500, mouse anti-CBX7 and anti- $\alpha$-Tubulin at 1:1000 dilutions. Gel bands were highlighted with the chemiluminescence detection kit (SuperSignal West Pico). Gel bands intensity was evaluated with ImageJ software Version 2.0.0-RC-43/1.51k.

\subsection{Chromatin Immunoprecipitation (ChiP) Assay}

ChiP assays on human specimens and cell line were carried out as already reported [5]. Samples were subjected to immunoprecipitation (IP) with the specific antibody against histone modification anti-tri methyl K9-Histone3, anti-tri methyl K4-Histone3, and anti-acetyl H3. qRT-PCR was performed as described [5].

\subsection{Statistical Analyses and Bioinformatics}

Data were statistically analyzed with two-tailed paired Student's t-test using Ka- 
leidaGraph 4.1.1 software and reported as means \pm standard deviation (SD). The significance was accepted at the level of $\mathrm{p}<0.05$.

UCSC (University of California Santa Cruz) genome browser was explored for H3K4me3. CBX7 binding sites were searched "on-line" using TFbind software [20] (Suehiro, Tsurumi, Yokohama, Japan), LASAGNA-Search 2.0 [21], and Alggen Promo Version 3.0.2 [22] [23]. OpenEpi Version 3.01 was used for power calculation.

\section{Results and Discussions}

\subsection{GKN1 Down-Regulation in GC Is Associated with Trimethylation of Histone 3 at Lysine 4 on the GKN1 Promoter}

In previous work, we investigated a possible epigenetic mechanism underlying the $G K N 1$ gene silencing in gastric carcinogenesis. We exemplified a functional model where a transcription factor might regulate the GKN1 promoter function by inducing histone methylation and deacetylation through SUV39H1 and HDACs (histone deacetylase), respectively and thus $G K N 1$ gene inactivation. In fact, chromatin immunoprecipitation assays for H3K9me3 showed that GKN1 down-regulation in gastric cancer (GC) tissues were associated with a high level of $\mathrm{H} 3 \mathrm{~K} 9 \mathrm{me} 3$. To highlight if also $\mathrm{H} 3 \mathrm{~K} 4 \mathrm{me} 3$ was associated with the epigenetic mechanism leading to the inactivation of $G K N 1$ gene, ChiP assays were performed. We chose to focus on H3K4me3 since together to H3K4me2, they are considered epigenetic marks linked with actively transcribed or transcriptionally poised genes [7] [24] [25]. As already reported, the experiment was carried out on three regions (A, B, and C) of the GKN1 promoter spacing for about $600 \mathrm{bp}$ [5] using two paired non-tumor $\left(\mathrm{N}_{1}-\mathrm{N}_{2}\right)$ and tumor $\left(\mathrm{T}_{1}-\mathrm{T}_{2}\right)$ specimens of gastric tissues taken from patients after surgery (Table 1). We first analyzed in these tissues the expression level of GKN1. Compared to non-tumor tissues, the expression of $G K N 1$ in the tumor tissues was completely absent (Figure 1A) whereas, a significant increase of $\mathrm{H} 3 \mathrm{~K} 9 \mathrm{me} 3$ in tumor samples was observed [5]. ChiP assays performed on the same GKN1 promoter regions (A, B, and C) revealed, compared with non-tumor tissues, an increase of H3K4me3 in $\mathrm{T}_{1}$ tumor tissue (Figure 1B) that was much less pronounced in sample $\mathrm{T}_{2}$ (Figure 1C). In fact, a very low value of $\mathrm{H} 3 \mathrm{~K} 4 \mathrm{me} 3$ enrichment and high values for the ChiP control samples were observed for sample $T_{2}$. These results somehow reflect some controversial aspects regarding the role of $\mathrm{H} 3 \mathrm{~K} 4 \mathrm{me} 3$ that is widely considered as an activating mark of transcription. In fact, evidence support that H3K4me3 is unlikely to be regulation of transcription initiation since its absence in the majority of cases seems to have no effect on nascent transcription [26]. The finding that the levels of H3K4 methylation could be a mark of chromatin that is kept in a "poised" state where is not actively transcribed, as also reported by Schneider et al., 2004 [7] in the case of inactive genes within the $\beta$-globin locus. To search for existing histone modification tracks, UCSC genome browser 
platforms were explored. No H3K4me3 was present in various cell lines in the region surrounding the $G K N 1$ transcription start site (TSS). Low level of $G K N 1$ mRNA and Pol2 and absence of H3K4me3 was instead observed in HeLa S3 clone despite the presence of an enhancer of H3K4 mono-methylated of about 1 kb upstream the GKN1 TSS.

One criticism of the present study regards the number of GC specimens used. Although the results obtained on two GC patients were roughly similar, to be confident that a mechanism underlying $G K N 1$ gene silencing is also based on the up-regulation of histone methylation $\mathrm{H} 3 \mathrm{~K} 4 \mathrm{me} 3$ by SUV39H1 and deacetylation mediated by HDAC1, a larger number of samples should be analyzed. This aspect is not easy to afford because of the difficulty in the recruitment of GC patients, in the quality and quantity of tissues specimens that can be obtained after surgery. Moreover, also the intrinsic difficulty of the ChiP assay experimental procedure should be taken into consideration.

To further investigate the epigenetic modifications on $G K N 1$ gene expression, we assayed the role of histone acetylation by using trichostatin A (TSA), an inhibitor of histone deacetylases (HDACs). As shown in Figure 2, ChiP assay performed on AGS gastric cancer cell line after treatment with TSA for $24 \mathrm{~h}$ showed a reduction of $\mathrm{H} 3 \mathrm{~K} 9 \mathrm{me} 3$ repressive modification (Figure $2 \mathrm{~A}$ ) and of $\mathrm{H} 3 \mathrm{~K} 4 \mathrm{me} 3$ (Figure 2B) that was associated by the increase of $\mathrm{H} 3$-acetylation-activating modification in the same three regions of the GKN1 promoter (Figure 2C). This result confirmed somehow the data obtained in human gastric tissues (Figure 1) and suggest that in this case $\mathrm{H} 3 \mathrm{~K} 4 \mathrm{me} 3$ is not associated to transcription since the treatment of gastric cancer cell line MKN28 with TSA resulted in an increase of GKN1 mRNA level [5].
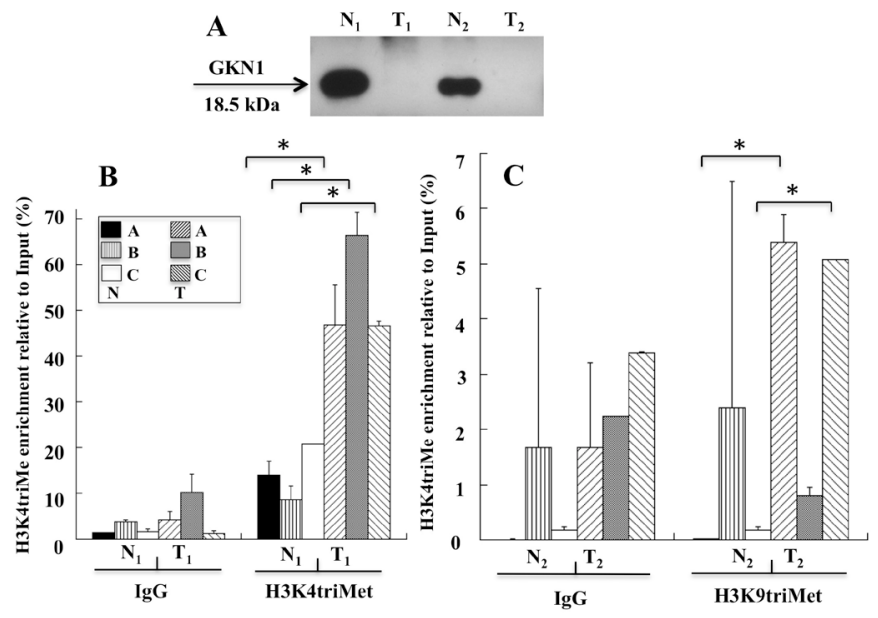

Figure 1. H3K4me3 levels on human GKN1 gene promoter. ChiP assays performed on human non-tumor $\left(\mathrm{N}_{1}-\mathrm{N}_{2}\right)$ and tumor $\left(\mathrm{T}_{1}-\mathrm{T}_{2}\right)$ human gastric samples, respectively. $\mathrm{H} 3 \mathrm{~K} 4 \mathrm{me} 3$ enrichment relative to input is reported as $2^{\Delta \mathrm{Ct}} \times 100$, where $\Delta \mathrm{Ct}$ is the difference between $\mathrm{Ct}_{\text {Input }}$ and $\mathrm{Ct}_{\mathrm{IP}}$. All quantitative ChiP data were derived from three independent experiments, and for each experiment, qPCR was performed in triplicate. ${ }^{\star} \mathrm{p}<$ 0.05 , compared to corresponding control. 

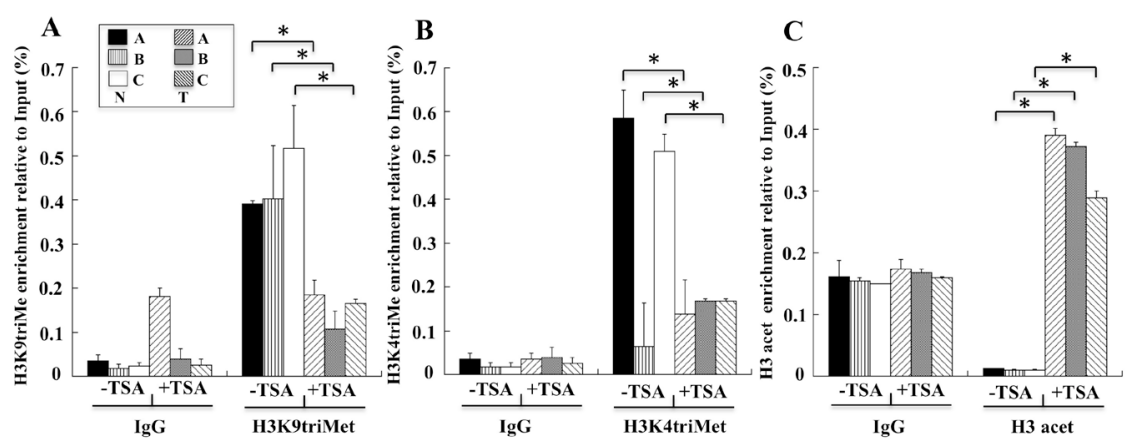

Figure 2. TSA induces in AGS cells decreased levels of H3K9me3, H3K4me3, and increased levels of $\mathrm{H} 3$ acetylation. Levels of $\mathrm{H} 3 \mathrm{~K} 9 \mathrm{me} 3$ (A), H3K4me3 (B), and H3 acetylation (C) determined by ChiP assays on AGS cells not treated (-TSA) and treated with TSA (+TSA). H3K9me3, H3K4me3, and $\mathrm{H} 3$ acetylation enrichment relative to input are reported as $2^{\Delta \mathrm{Ct}} \times 100$, where $\Delta \mathrm{Ct}$ is the difference between $\mathrm{Ct}_{\text {Input }}$ and $\mathrm{Ct}_{\mathrm{IP}}$. All quantitative ChiP data were derived from three independent experiments, and for each experiment, qRT-PCR was performed in triplicate. ${ }^{\star} \mathrm{p}<0.05$, compared to corresponding control.

Table 1. Clinical and histopathological characteristics of gastric cancer patients*

\begin{tabular}{cc}
\hline Variable & n. 9 \\
\hline Age at Surgery (yrs) & $64.5 \pm 9.6$ \\
Mean \pm SD & $53-78$ \\
Range & $7 / 2$ \\
Sex M/F & \\
Tumor Type & $4\left(\mathrm{P}_{1,3,4,8}\right)$ \\
Intestinal & $5\left(\mathrm{P}_{2,5,6,7,9)}\right.$ \\
Diffuse & \\
Stomach Region & 3 \\
Antrum & 6 \\
Corpus-Fundus & \\
Grade of Differentiation & $2(\%)$ \\
Moderate & $7(\%)$ \\
Poor & \\
Stage & $0(0 \%)$ \\
Early & $9(100 \%)$ \\
Advanced &
\end{tabular}

${ }^{*}$ Non-tumor $\left(\mathrm{N}_{3}-\mathrm{N}_{7}\right)$ and tumor $\left(\mathrm{T}_{3}-\mathrm{T}_{7}\right)$ specimens reported in Figure 3 were already used in previous work to analyze the $G K N 1$ expression levels (Di Stadio et al., 2017). $\mathrm{P}=$ Patients.

\subsection{CBX7 and GKN1 Expression Levels in GC Non-Tumor and Tumor Tissues}

In previous work, we showed that RUNX3 was not a possible transcription factor functioning as a negative regulator able to induce $G K N 1$ gene inactivation. Because CBX7 is reported to be overexpressed in gastric tumors [16], we tried to highlight if $\mathrm{CBX} 7$ could be the specific transcription factor able to bind $G K N 1$ promoter and thus to promote the formation of a multifactor complex including 
SUV39H1 and/or other histone modifier enzymes involved in GKN1 down-regulation during gastric carcinogenesis. Therefore, we analyzed by Western blot the expression level of CBX7 in a collection of paired non-tumor and tumor specimens from patients with gastric cancer during surgery (Table 1). The down-expression of GKN1 observed in gastric tumor tissues (Figure 3) was not always accompanied by the up-regulation of CBX7. This observation was established from the Western blot band intensity ratio (Figure 3) of non-tumor tissue and that of the corresponding tumor one minus 1 (N/T-1) (Figure 4). However, the intensity values evaluated with the Image J software represent only a trend since they were not statistically significant. This aspect was also confirmed by the power calculation of the average difference between the two populations ( $G K N 1$, and CBX7 band intensities). With a power of $80 \%$ at $95 \%$ confidence level, the sample size required was 301 . Moreover, the calculated coefficient of variation (38.7\% for $G K N 1$, and $34.1 \%$ for $\mathrm{CBX} 7)$ indicated a large biological variability within each group.

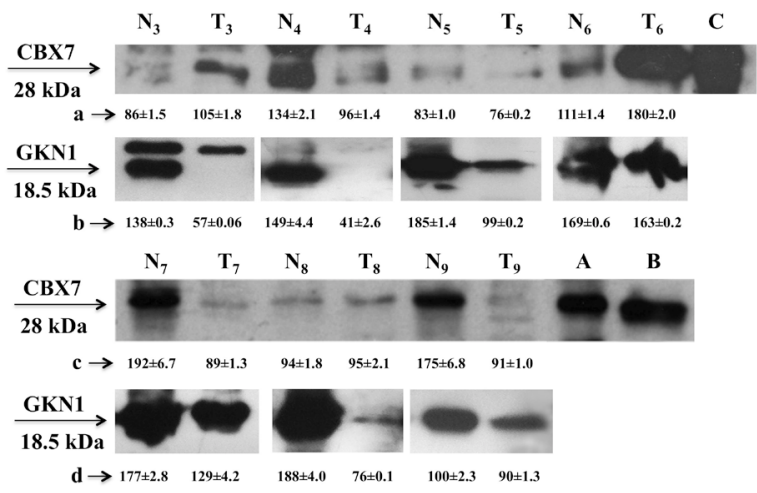

Figure 3. CBX7 and GKN1 expression levels in human gastric tissues. The expression levels of CBX7 in non-tumor $\left(\mathrm{N}_{3}-\mathrm{N}_{9}\right)$ and tumor $\left(\mathrm{T}_{3}-\mathrm{T}_{9}\right)$ paired gastric samples and corresponding expression levels of $G K N 1$ were evaluated with ImageJ software. (A) AGS gastric cancer cell line. (B) Normal gastric mucosa. (a, b, c, d) average band intensity \pm SD of three measurements. SD: Standard deviation.

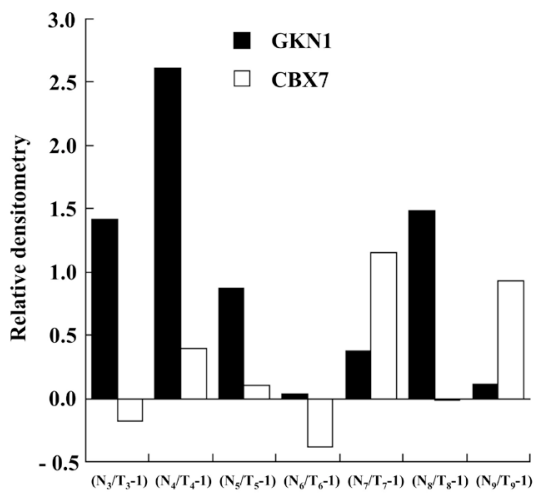

Figure 4. Comparison between $G K N 1$ and CBX7 expression levels of in the tumor tissues analyzed. The relative expression of GKN1 and CBX7 was determined from the band intensity ratio $(\mathrm{N} / \mathrm{T})$ of non-tumor tissue and that of the corresponding tumor one, minus $1(\mathrm{~N} / \mathrm{T}-1)$. 


\section{Conclusion}

In present study, we addressed the role of $\mathrm{H} 3 \mathrm{~K} 4 \mathrm{me} 2$ as a possible epigenetic marker involved in $G K N 1$ gene silencing in gastric carcinogenesis. Our findings, and in particular those observed for sample $\mathrm{N}_{1} / \mathrm{T}_{1}$ and for AGS cells, as opposed to the proposed role of the $\mathrm{K} 4$ trimethylation of histone $\mathrm{H} 3$ as a transcription activator, reinforces the controversial hypothesis that its deposition is transcription independent. Moreover, our experimental evidence showed that compared to non-tumor tissues, the expression of CBX7 in tumor gastric tissues was not always overexpressed and thus associated with the down-regulation of GKN1. ChiP assay might clarify the possible binding of $\mathrm{CBX} 7$ on the $G K N 1$ promoter since bioinformatic tools like TFbind, LASAGNA and PROMO did not highlight CBX7 consensus binding sequence on GKN1 promoter. In particular, PROMO home page predicted a large number of possible transcription factors but dissimilarity margin less or equal to $15 \%$. The result strongly suggests the non-involvement of the transcription factor in the regulation of $G K N 1$ silencing in gastric cancer.

\section{Acknowledgements}

The authors thank Dr. G. de Dominicis, Hospital Cardarelli, Naples, Italy, for tissue histopathological classification and immunohistochemistry. This work was supported by funds from Programmi di Ricerca Scientifica di Rilevante Interesse Nazionale (2012CK5RPF_004), PON Ricerca e Competitività 2007-2013 (PON01_02782) and POR Campania FSE 2007-2013, Project CRÈME.

\section{Authors' Contributions}

CSDS and FA were involved in ChiP assays, AF performed qRT-PCR analysis and statistical evaluation, GM was involved in $\mathrm{WB}$ and cell extract preparation, MG takes care of patient recruitment, ER was involved in the preparation of a draft manuscript, PA in the reading and approval of the final manuscript.

\section{Conflicts of Interest}

The authors declare no conflicts of interest regarding the publication of this paper.

\section{References}

[1] Oien, K.A., McGregor, F., Butler, S., Ferrier, R.K., Downie, I., Bryce, S., Burns, S. and Keith, W.N. (2004) Gastrokine 1 Is Abundantly and Specifically Expressed in Superficial Gastric Epithelium, Down-Regulated in Gastric Carcinoma, and Shows High Evolutionary Conservation. The Journal of Pathology, 203, 789-797. https://doi.org/10.1002/path.1583

[2] Nardone, G., Martin, G., Rocco, A., Rippa, E., La Monica, G., Caruso, F. and Arcari, P. (2008) Molecular Expression of Gastrokine 1 in Normal Mucosa and in Helicobacter pylori-Related Preneoplastic and Neoplastic Gastric Lesions. Cancer Biology 
and Therapy, 7, 1890-1895. https://doi.org/10.4161/cbt.7.12.6936

[3] Rippa, E., La Monica, G., Allocca, R., Romano, M.F., De Palma, M. and Arcari, P. (2011) Overexpression of Gastrokine 1 in Gastric Cancer Cells Induces Fas-Mediated Apoptosis. Journal of Cellular Physiology, 226, 2571-2578.

https://doi.org/10.1002/jcp.22601

[4] Pavone, L.M.N., Del Vecchio, P., Mallardo, P., Altieri, F., De Pasquale, V., Rea, S., Martucci, N.M., Di Stadio, C.S., Pucci, P., Flagiello, A., Masullo, M., Arcari, P. and Rippa, E. (2013) Structural Characterization and Biological Properties of Human Gastrokine 1. Molecular BioSystems, 9, 412-421. https://doi.org/10.1039/c2mb25308a

[5] Altieri, F., Di Stadio, C.S., Federico, A., Miselli, G., De Palma, M., Rippa, E. and Arcari, P. (2017) Epigenetic Alterations of Gastrokine 1 Gene Expression in Gastric Cancer. Oncotarget, 810, 16899-16911. https://doi.org/10.18632/oncotarget.14817

[6] Bernstein, B.E., Humphrey, E.L., Erlich, R.L., Schneider, R., Bouman, P., Liu, J.S., Kouzarides, T. and Schreiber, S.L. (2002) Methylation of Histone H3 Lys 4 in Coding Regions of Active Genes. Proceedings of the National Academy of Sciences of the United States of America, 99, 8695-8700. https://doi.org/10.1073/pnas.082249499

[7] Schneider, R., Bannister, A.J., Myers, F.A., Thorne, A.W., Crane-Robinson, C. and Kouzarides, T. (2004) Histone H3 Lysine 4 Methylation Patterns in Higher Eukaryotic Genes. Nature Cell Biology, 6, 73-77. https://doi.org/10.1038/ncb1076

[8] Bannister, A.J., Zegerman, P., Partridge, J.F., Miska, E.A., Thomas, J.O., Allshire, R.C. and Kouzarides, T. (2001) Selective Recognition of Methylated Lysine 9 on Histone H3 by the HP1 Chromo Domain. Nature, 410, 120-124.

https://doi.org/10.1038/35065138

[9] Stewart, M.D., Li, J. and Wong, J. (2005) Relationship between Histone H3 Lysine 9 Methylation, Transcription Repression, and Heterochromatin Protein 1 Recruitment. Molecular and Cellular Biology, 25, 2525-2538. https://doi.org/10.1128/MCB.25.7.2525-2538.2005

[10] Di Croce, L. and Helin, K. (2013) Transcriptional Regulation by Polycomb Group Proteins. Nature Structural and Molecular Biology, 20, 1147-1155. https://doi.org/10.1038/nsmb.2669

[11] Pallante, P., Federico, A., Berlingieri, M.T., Bianco, M., Ferraro, A., Forzati, F., Iaccarino, A., Russo, M., Pierantoni, G.M., Leone, V., Sacchetti, S., Troncone, G., Santoro, M. and Fusco, A. (2008) Loss of the CBX7 Gene Expression Correlates with a Highly Malignant Phenotype in Thyroid Cancer. Cancer Research, 68, 6770-6778. https://doi.org/10.1158/0008-5472.CAN-08-0695

[12] Pallante, P., Terracciano, L., Carafa, V., Schneider, S., Zlobec, I., Lugli, A., Bianco, M., Ferraro, A., Sacchetti, S., Troncone, G., Fusco, A. and Tornillo, L. (2010) The Loss of the CBX7 Gene Expression Represents an Adverse Prognostic Marker for Survival of Colon Carcinoma Patients. European Journal of Cancer, 46, 2304-2313. https://doi.org/10.1016/j.ejca.2010.05.011

[13] Karamitopoulou, E., Pallante, P., Zlobec, I., Tornillo, L., Carafa, V., Schaffner, T., Borner, M., Diamantis, I., Esposito, F., Brunner, T., Zimmermann, A., Federico, A., Terracciano, L. and Fusco, A. (2010) Loss of the CBX7 Protein Expression Correlates with a More Aggressive Phenotype in Pancreatic Cancer. European Journal of Cancer, 46, 1438-1444. https://doi.org/10.1016/j.ejca.2010.01.033

[14] Zhang, X.W., Zhang, L., Qin, W., Yao, X.H., Zheng, L.Z., Liu, X., Li, J. and Guo, W.J. (2010) Oncogenic Role of the Chromobox Protein CBX7 in Gastric Cancer. 
Journal of Experimental and Clinical Cancer Research, 29, Article ID: 4320. https://doi.org/10.1186/1756-9966-29-114

[15] Forzati, F., Federico, A., Pallante, P., Fedele, M. and Fusco, A. (2012) Tumor Suppressor Activity of CBX7 in Lung Carcinogenesis. Cell Cycle, 11, 1888-1891. https://doi.org/10.4161/cc.20022

[16] Ni, S.-J., Zhao, L.-Q., Wang, X.-F., Wu, Z.-H., Hua, R.-X., Wan, C.-H., Zhang, J.-Y., Zhang, X.-W., Huang, M.-Z., Gan, L., Sun, H.-L., Dimri, G.P. and Guo, W.-J. (2018) CBX7 Regulates Stem Cell-Like Properties of Gastric Cancer Cells via p16 and AKT-NF- $\kappa$ BmiR-21 Pathways. Journal of Hematology and Oncology, 11, Article ID: 1276. https://doi.org/10.1186/s13045-018-0562-Z

[17] Di Stadio, C.S., Altieri, F., Federico, A., Miselli, G., Niglio, A., De Palma, M., Rippa, E. and Arcari, P. (2017) Down-Regulation of GKN1 in Gastric Cancer Is Not Associated with the RUNX3 Expression. Journal of Biosciences and Medicines, 5, 1-11. https://doi.org/10.4236/jbm.2017.511009

[18] Di Stadio, C.S., Altieri, F., Miselli, G., Elce, A., Severino, V., Chambery, A., Quagliariello, V., Villano, V., de Dominicis, G., Rippa, E. and Arcari, P. (2016) AMP18 Interacts with the Anion Exchanger SLC26A3 and Enhances Its Expression in Gastric Cancer Cells. Biochimie, 121, 151-160. https://doi.org/10.1016/j.biochi.2015.12.010

[19] Edge, S.B. and Compton, C.C. (2010) The American Joint Committee on Cancer: The 7th Edition of the AJCC Cancer Staging Manual and the Future of TNM. Annals of Surgical Oncology, 17, 1471-1474. https://doi.org/10.1245/s10434-010-0985-4

[20] Tsunoda, T. and Takagi, T. (1999) Estimating Transcription Factor Bindability on DNA. Bioinformatics, 15, 622-630. https://doi.org/10.1093/bioinformatics/15.7.622

[21] Lee, C. and Huang, C.H. (2013) LASAGNA-Search: An Integrated Web Tool for Transcription Factor Binding Site Search and Visualization. BioTechniques, 54, 141-153. https://doi.org/10.2144/000113999

[22] Messeguer, X., Escudero, R., Farré, D., Nuñez, O., Martínez, J. and Albà, M.M. (2002) PROMO: Detection of Known Transcription Regulatory Elements Using Species-Tailored Searches. Bioinformatics, 18, 333-334. https://doi.org/10.1093/bioinformatics/18.2.333

[23] Farré, D., Roset, R., Huerta, M., Adsuara, J.E., Roselló, L., Albà, M.M. and Messeguer, X. (2003) Identification of Patterns in Biological Sequences at the ALGGEN Server: PROMO and MALGEN. Nucleic Acids Research, 31, 3651-3653. https://doi.org/10.1093/nar/gkg605

[24] Cruz, C., Rosa, D.M., Krueger, C., Gao, Q., Horkai, D., King. M., Field, M. and Houseley, J. (2018) Trimethylation of Histone H3 Lysine 4 Facilitates Gene Expression in Ageing Cells. eLife, 7, e34081.

[25] Santos-Rosa, H., Schneider, R., Bannister, A.J., Sherriff, J., Bernstein, B.E., Emre, N.C., Schreiber, S.L., Mellor, J. and Kouzarides, T. (2002) Active Genes Are Tri-Methylated at K4 of Histone H3. Nature, 419, 407-411. https://doi.org/10.1038/nature01080

[26] Howe, F.S., Fischl, H., Murray, S.C. and Mellor, J. (2016) Is H3K4me3 Instructive for Transcription Activation? Bioessays, 39, 1-12. 


\section{Abbreviations}

ChiP: Chromatin Immunoprecipitation;

GC: Gastric Cancer;

GKN1: Gastrokine 1;

HDAC: Histone Deacetylase;

H3K4me3: Trimethylation of Histone 3 at Lysine 4;

H3K9me3: Trimethylation of Histone 3 at Lysine 9;

TSA: Trichostatin A;

TSS: Transcription Start Site;

SUV39H1: Histone-Lysine N-Methyltransferase. 